Bulletin of Pharmaceutical Sciences
Assiut University
Website: http://bpsa.journals.ekb.eg/
e-mail: bullpharm@aun.edu.eg

\title{
ROSIGLITAZONE SHOWS SEQUENCE-SPECIFIC SYNERGY WITH CHEMOTHERAPEUTIC DRUGS IN INHIBITION OF MCF-7 BREAST CANCER CELL LINE
}

\author{
Sally Al-Moualem ${ }^{1 *}$ and Fawza Monem ${ }^{1,2}$ \\ ${ }^{1}$ Program of Molecular Biology and Biotechnology, Department of Biochemistry and \\ Microbiology, Faculty of Pharmacy, Damascus University, Damascus, Syria \\ ${ }^{2}$ Department of Clinical Laboratories, Al-Assad Hospital, Damascus, Syria
}

\begin{abstract}
Preclinical studies have shown that peroxisome proliferator-activated receptor $\gamma(P P A R \gamma)$ ligands such as thiazolidinediones (TZDs) can exert antitumor effects against breast cancer and a variety of other cancers. In this study, we investigated the potential of repurposing a PPAR $\gamma$ ligand, rosiglitazone (RGZ), in combination with either of three chemotherapeutic agents, doxorubicin (Dox) or cisplatin (Cis) or 5-fluorouracil (5-FU), for the in-vitro treatment of breast cancer cell line, MCF-7. RGZ augmented the growth inhibition effect of Cis and 5-FU on MCF7 cells. The synergy was observed only when chemotherapy preceded RGZ and not vice versa, demonstrating a sequence-specific effect. We also observed that the first administered drug gave its cell cycle pattern and apoptosis/necrosis pattern to the subsequently applied drug. Besides, no adipose differentiation in the form of lipid droplet accumulation was induced in treated cells regardless of the used drugs and their sequence of application. Together, our data show a synergistic effect of administering RGZ after Cis or 5-FU and suggests an inhibitory role of RGZ on the chemo-resistant MCF-7 side-populations.
\end{abstract}

\section{INTRODUCTION}

Differentiation therapy is defined as the reactivation of normal differentiation pathways in tumor cells to achieve a less aggressive, more tolerable phenotype to traditional adopted medications ${ }^{1}$. Solid tumors are characterized by their heterogeneity in differentiation state and mitotic potential. While the normal tissue differentiation process includes cellular growth or arrest, many malignant cells fail to express adequate levels of molecular or morphological markers of the terminally-differentiated state, which makes these cells more resistant to chemo- and radiotherapy. Thus, differentiation therapy could induce the development of a tumoral phenotype with better prognosis ${ }^{2 \& 3}$.

Drug repurposing or repositioning is the process of seeking new medical treatments within the drugs available with a particular medical indication, such as hypertension, diabetes, or hyperlipidemia, instead of developing drugs de novo. It is based on the principle of polypharmacology, where one drug with multiple target and off-target effects may present various modes of action. Enthusiasm for drug repurposing is growing, especially in the field of oncology, where common examples include statins, angiotensin-receptor blockers, metformin, aspirin, and vitamin $\mathrm{D}^{4 \& 5}$.

PPAR $\gamma s$ are transcription factors belonging to the nuclear receptors family and involved in many pathways, including adipogenesis, apoptosis, and cell cycle. These receptors are activated by binding to their specific ligands. They undergo morphological alterations, form heterodimers with the retinoid $\mathrm{X}$ receptor (RXR), and bind to particular PPAR $\gamma$ response elements (PPREs) in many genes' regulating regions ${ }^{6}$. PPAR $\gamma$ ligands of thiazolidinediones (TZDs) family (FDA approved for the treatment of Type 2 Diabetes) 
showed effectiveness in proliferation and invasion inhibition and apoptosis in multiple tumors, including breast cancer, lung cancer and leukemia ${ }^{7 \& 8}$.

There is some evidence on the use of TZDs in the treatment of breast cancer by inducing apoptosis, cell cycle arrest, autophagy, and differentiation and by reducing angiogenesis and metastasis ${ }^{9 \& 10}$. Mueller et al. $^{2}$ reported a significant increase in the gene expression of PPAR $\gamma$ in the majority of human metastatic breast carcinomas. Activation of PPAR $\gamma$ leads to the accumulation of lipids and alterations of gene expression, including upregulation of a normal breast development biomarker, maspin, which has anti-tumoral activity.

In addition to evidence supporting the possibility that PPAR $\gamma$ activation will become one of the strategies used in treating breast cancer; its relatively low toxicity and good clinical tolerance could be promising in its use in chemoprevention or post-surgical supportive treatment ${ }^{2}$.

Breast cancer is the most common cancer among females, affecting approximately 2.1 million women yearly worldwide ${ }^{11}$. Although chemotherapy has dramatically improved patients' survival in recent decades, the chemoresistance phenomenon remains one of the significant challenges in cancer treatment worldwide and is responsible for tumor recurrence. Hence, we aimed to assess the effect of combining differentiation therapy (PPAR $\gamma$ agonist) with traditional chemotherapy on cell growth, apoptosis, and differentiation of the breast cancer cell line MCF-7.

\section{MATERIALS AND METHODS}

\section{Materials}

The MCF-7 cell line was obtained from The European Collection of Authenticated Cell Cultures (ECACC), Sigma-Aldrich (USA). Roswell Park Memorial Institute (RPMI)-1640 Medium and penicillin-streptomycin antibiotics were obtained from EuroClone (Italy). Fetal bovine serum (FBS) was obtained from SigmaAldrich (USA) and recombinant human insulin from Novo Nordisk (Denmark). MTT reagent and cell culture grade dimethyl sulfoxide (DMSO) were obtained from Genaxxon (Germany). Doxorubicin and 5-fluorouracil from Ebewe (Austria) and cisplatin from Mylan (France).

\section{Cell culture and treatment}

The MCF-7 cell line was maintained in a complete medium of RPMI-1640 supplemented with $10 \%$ fetal bovine serum, $1 \%$ recombinant human insulin, and $1 \%$ penicillin-streptomycin antibiotics. Cells were incubated at $37^{\circ} \mathrm{C}$ with $5 \% \mathrm{CO} 2$, and the medium was replaced every 48 to 72 hours. Rosiglitazone (RGZ) dissolved in DMSO, and doxorubicin (Dox), 5fluorouracil (5-FU), and cisplatin (Cis) aqueous solutions were used in drug sensitivity assays.

For drug combinations, cells were cultured with the complete medium in 96-well plates. On the next day, cells were treated in the presence of serum with RGZ at its halfmaximal inhibitory concentration $\left(\mathrm{IC}_{50}\right), 50$ $\mu \mathrm{M}$, for 48 hours, followed by 24 hours of drug-free medium or 4 hours of drug-free medium followed by 20 hours of treatment with the chemotherapeutic agent. In other wells, cells were treated with the chemotherapeutic agent (Dox or Cis or 5-FU) for 20 hours followed either by 52 hours of drug-free medium or by 4 hours of drug-free medium followed by 48 hours of treatment with RGZ $(50 \mu \mathrm{M})$, (Fig. 1). Cell growth was measured using the 3-(4,5-dimethylthiazol-2-yl)-2,5diphenyltetrazolium bromide (MTT) assay 72 hours after treatment initiation.

\section{Measurement of cell growth}

Cell growth was measured using the tetrazolium salt (MTT), which is cleaved by a mitochondrial dehydrogenase to produce dark blue formazan crystals. The crystals were dissolved using DMSO. Optical density was measured at $540 \mathrm{~nm}$, and the approximate livecell number was determined from the cell line standard curve (cell density-optical density). Two independent experiments were performed in six replicates, and the mean value was calculated for each condition, and cell growth calculated as:

$$
\begin{aligned}
& \text { Percentage of cell growth }= \\
& \frac{\text { Mean Cell No.in treated wells }}{\text { Mean Cell No.in control wells }} \times 100
\end{aligned}
$$




\section{Median-effect analysis (MEA)}

The median-effect analysis was performed as described by Chou and Talalay ${ }^{12}$. CompuSyn software $^{13}$ was used to calculate combination indices (CIs) for augmented concentrations of chemotherapeutic drugs combined with a fixed concentration of RGZ and average CIs. A CI of 1 indicates an additive effect, whereas a $\mathrm{CI}<1$ indicates synergy, and $\mathrm{CI}>1$ indicates antagonism.

\section{Flow cytometry}

At 72-h post-treatment, the cells were detached using trypsin-EDTA and washed twice with PBS, then resuspended in serumfree medium. For the Apoptosis/Necrosis assay, cells were stained using Annexin-V$\mathrm{GFP}^{14}$ (Atomic Energy Commission of Syria (AECS), Syria) and propidium iodide (PI) and analyzed using BD FACSCalibur Flow
Cytometer (BD Biosciences, San Jose, CA). For cell cycle analysis, the DNA content of PIstained cells was analyzed by flow cytometer as described by Darzynkiewicz et al. ${ }^{15}$.

\section{Nile blue staining}

Cells were cultured with the complete medium in 12-well plates and treated as described in figure 1. After 72 hours, wells were washed twice using PBS and fixed with paraformaldehyde (4\% in PBS) for 30 minutes. Cells were washed 2 to 3 times with PBS and stained with Nile blue solution $(1 \mu \mathrm{g} / \mathrm{ml}$ in distilled water) for 20 minutes in the dark. Plates were rinsed with distilled water and examined using a fluorescent microscope (Nikon Eclipse TS100, Japan). Oleic acid (1/4000) for 24 hours was used as a positive control.

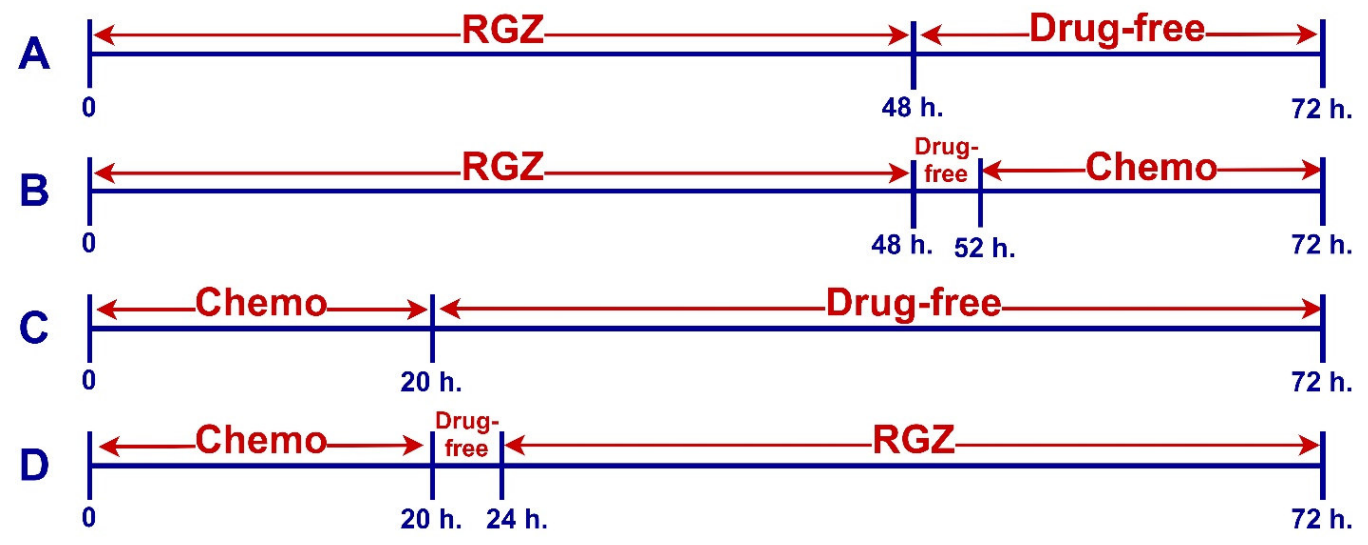

Fig. 1: Timeline of applied drugs (Chemotherapy and rosiglitazone) on MCF-7 cell line: (A) in studies of rosiglitazone (RGZ) as a single agent, RGZ was administered for 48 hours followed by 24 hours without drug; (B) in studies of RGZ before treatment, cells were treated with RGZ for 48 hours followed by a 4hours drug-free washout and Dox or Cis or 5-FU for 20 hours; (C) in studies of single-agent chemotherapy, each chemotherapeutic drug (doxorubicin (Dox) or cisplatin (Cis) or 5-fluorouracil (5-FU)) was administered for 20 hours followed by 52 hours without drug; (D) in studies of RGZ after treatment, cells were treated with Dox, Cis or 5-FU for 20 hours followed by a 4-hour drug-free washout and RGZ for 48 hours. In single-agent studies, cells were treated with RGZ at concentrations of 12.5, 25, 50, 100, 200 $\mu \mathrm{M}$, Dox at concentrations of $0.25,0.5,1$ or $2 \mu \mathrm{M}$, Cis at concentrations of $12.5,25,50$ or $100 \mu \mathrm{g} / \mathrm{ml}$ and 5 -FU at concentrations of $50,100,200$ or $400 \mu \mathrm{g} / \mathrm{ml}$. In combination studies, cells were treated with $50 \mu \mathrm{M} \mathrm{RGZ}$ before or after various concentrations of Dox or Cis or 5-FU as indicated. 


\section{RESULTS AND DISCUSSION}

\section{Results}

RGZ potentiates Cis- or 5-FU-induced MCF-7 growth inhibition in a sequencespecific manner

To assess the efficacy of combining rosiglitazone with some cytotoxic chemotherapeutic drugs against breast cancer cells, we applied previously mentioned drugs in a sequence-specific manner and reported cell growth after treatment. We found that cell growth inhibition was the highest when MCF-7 cells were treated with Dox or 5-FU or Cis followed by RGZ, noting that the application of RGZ after Dox resulted in significant inhibition of cell growth compared with Dox alone, only at smaller Dox concentrations.

We observed similar results when administering combinations of $\mathrm{Cis}$ or $5-\mathrm{FU}$ with RGZ, where we found sequence-specific effect potentiation appearing when RGZ followed Cis or 5-FU. However, RGZ blocked the growth inhibitory effect of the subsequent chemotherapeutic drug (Cis, Dox, and 5-FU) independently of chemotherapy type, its mechanism of action, or its concentration, as we noticed a fluctuation in the percentage of cell viability around the $\mathrm{IC}_{50}$ of $\mathrm{RGZ}$ $(48.42 \pm 2.10 \mu \mathrm{M})$ in wells treated with RGZ followed by chemotherapy: Dox, 5-FU and Cis (55.4 $\pm 9.34 \mu \mathrm{M}),(p=0.13338)$ (Fig. 2).

Median-effect analysis revealed sequencespecific synergy when RGZ followed Cis or 5-FU

RGZ-chemotherapy interactions were assessed using median-effect analysis. The average CI for the combination of Cis followed by RGZ is 0.75 . Conversely, with the combination where RGZ was followed by Cis, where average CI was 4.43 , indicating partial antagonism. MEA revealed synergy between Dox and RGZ when applying Dox followed by RGZ, only at low Dox concentration. On the other hand, no growth inhibition or synergy approved when using higher Dox concentrations.

Similarly, we found a sequence-specific synergy when applying 5-FU followed by RGZ (average $\mathrm{CI}=0.56$ ) but partial antagonism when 5 -FU was preceded by RGZ (average $\mathrm{CI}=$ 5.48) (Fig. 3).

\section{RGZ following Cis shifted cells from early to late apoptosis}

To determine cell percentage in early or late apoptosis in treated cell cultures, cell analysis was performed using PI and AnnexinV- GFP and flow cytometer. Live cells remained uncolored (Annexin-V-GFP/PI'). Cells in early apoptosis showed Annexin-VGFP positive and PI negative staining patterns (Annexin-V-GFP ${ }^{+} / \mathrm{PI}^{-}$), whereas cells in late apoptosis showed Annexin V and a PI-positive staining pattern (Annexin-V-GFP ${ }^{+} / \mathrm{PI}^{+}$). Finally, Necrotic cells showed PI-only staining pattern (Annexin-V-GFP $/ \mathrm{PI}^{+}$).

In control non-treated cell cultures, $96.3 \%$ of MCF-7 were Annexin-V-GFP'/PI', $2.6 \%$ were Annexin-V-GFP ${ }^{+} / \mathrm{PI}^{-}$and $1.1 \%$ were Annexin-V-GFP $/ \mathrm{PI}^{+}$with no necrotic cells. RGZ treated cells $(50 \mu \mathrm{M}), 48$ hours showed similar staining patterns to control cells, with 91.5\% (Annexin-V-GFP $/ \mathrm{PI}^{-}$), 3.96\% (Annexin$\mathrm{V}-\mathrm{GFP}^{+} / \mathrm{PI}^{-}$), and $4.5 \%$ (Annexin-V-GFP $/ \mathrm{PI}^{+}$).

Application of RGZ $(50 \mu \mathrm{M})$ preceding Cis resulted in an increase of apoptotic cells (33.1\% and $9.5 \%$ ) compared with control cells (2.6\% and $1.1 \%)$. Conversely, Cis treatment $(25 \mu \mathrm{g} / \mathrm{ml})$ resulted in $0.4 \%$ live cells, $73.9 \%$ early apoptotic cells, $25.8 \%$ late apoptotic and $0.01 \%$ necrotic cells. Application of Cis followed by RGZ $(50 \mu \mathrm{M})$ resulted in a rise in apoptotic cell percentage (52.4\% and $47.3 \%$ ) compared with control and RGZ followed by Cis treated cells, with the observation of cell shift to late apoptosis pattern compared with early apoptotic pattern in cells treated with Cis alone.

Dox treatment $(1 \mu \mathrm{M}, 24 \mathrm{~h}$.$) exhibited a$ decrease in live-cell percentage to $30.4 \%$ and an increase in early apoptotic (36.3\%) and late apoptotic cells $(27.1 \%)$ and necrotic cells proportions $(6.6 \%)$. This pattern did not change when treating cells with RGZ $(50 \mu \mathrm{M}, 24$ h.) after Dox. Conversely, the application of Dox after RGZ caused a little decrease in cell viability $(85.5 \%)$ with an increase in necrosis (7\%) compared with control and RGZ-treated cells (Fig. 4). 

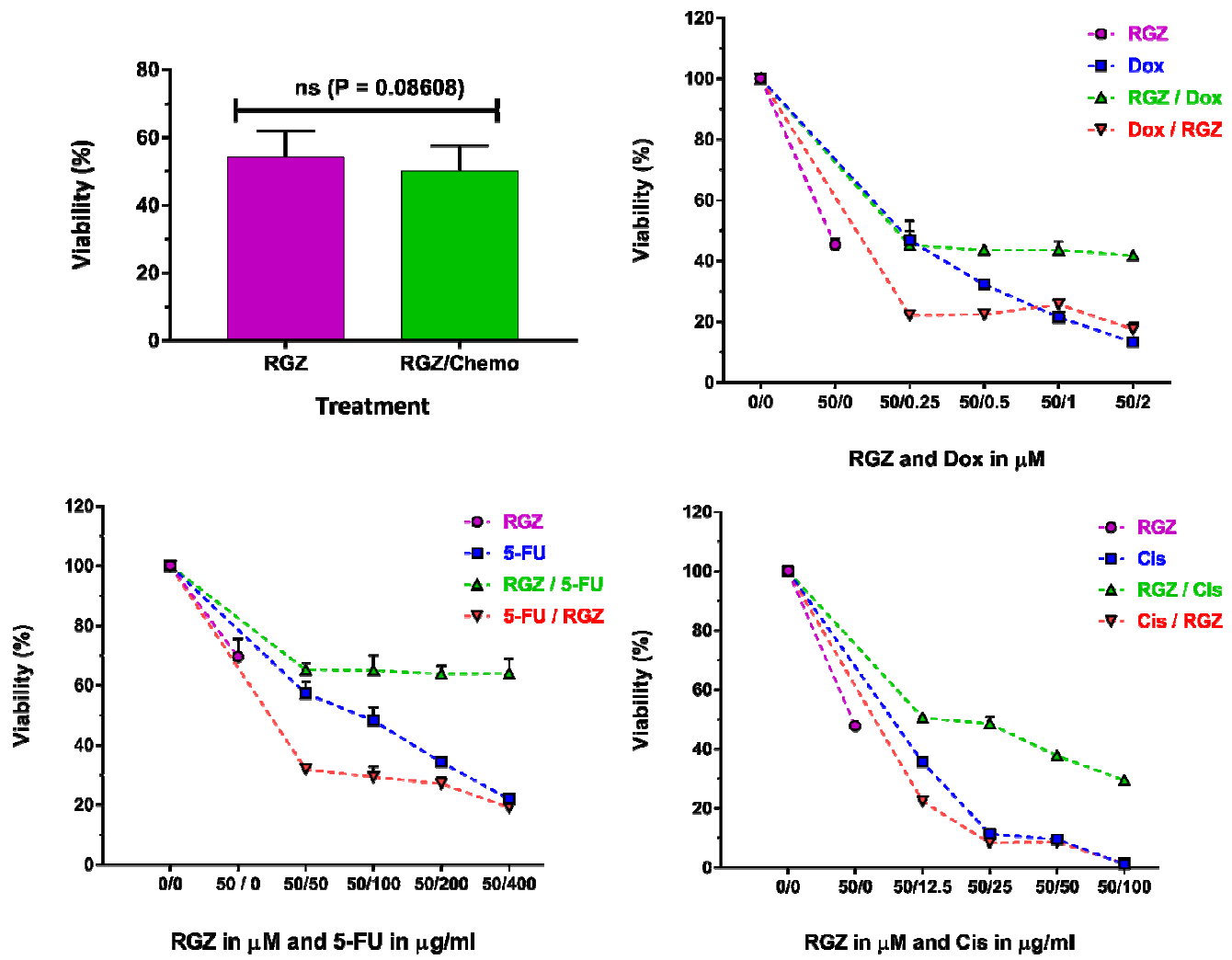

Fig. 2: Sequence-specific interaction between rosiglitazone (RGZ) and either of doxorubicin (Dox), or cisplatin (Cis), or 5-fluorouracil (5-FU) in growth inhibition of MCF-7 cells. (A) MCF-7 growth inhibition comparison between wells treated with RGZ and wells treated with RGZ followed by chemotherapy. (B) MCF-7 cell growth kinetics in various combinations of RGZ and Dox; (C) of RGZ and 5-FU; and (D) of RGZ and Cis.

A

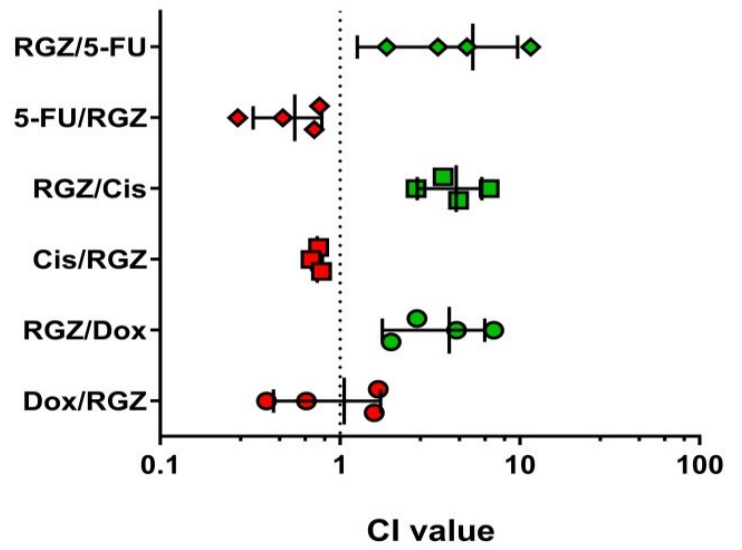

B

\begin{tabular}{|c|c|c|}
\hline \hline Combination & Average CI & Interaction \\
\hline RGZ/Dox & 4.05 & Antagonism \\
\hline Dox/RGZ & 1.06 & Near-additive \\
\hline RGZ/Cis & 4.43 & Antagonism \\
\hline Cis/RGZ & 0.75 & Synergy \\
\hline RGZ/5-FU & 5.48 & Antagonism \\
\hline 5-FU/RGZ & 0.56 & Synergy \\
\hline \hline
\end{tabular}

Fig. 3: Combination indices (CIs) for different combinations (rosiglitazone-chemotherapy), calculated using CompuSyn software: (A) Scatter dot plot showing combination index (CI) values for each drug combination; and (B) table showing average CI for each tested combination. A CI of 1 indicates additive effects, whereas a $\mathrm{CI}<1$ indicates synergy, and $\mathrm{CI}>1$ indicates antagonism. Cis: cisplatin, Dox: doxorubicin, 5-FU: 5-fluorouracil, and RGZ: rosiglitazone. 


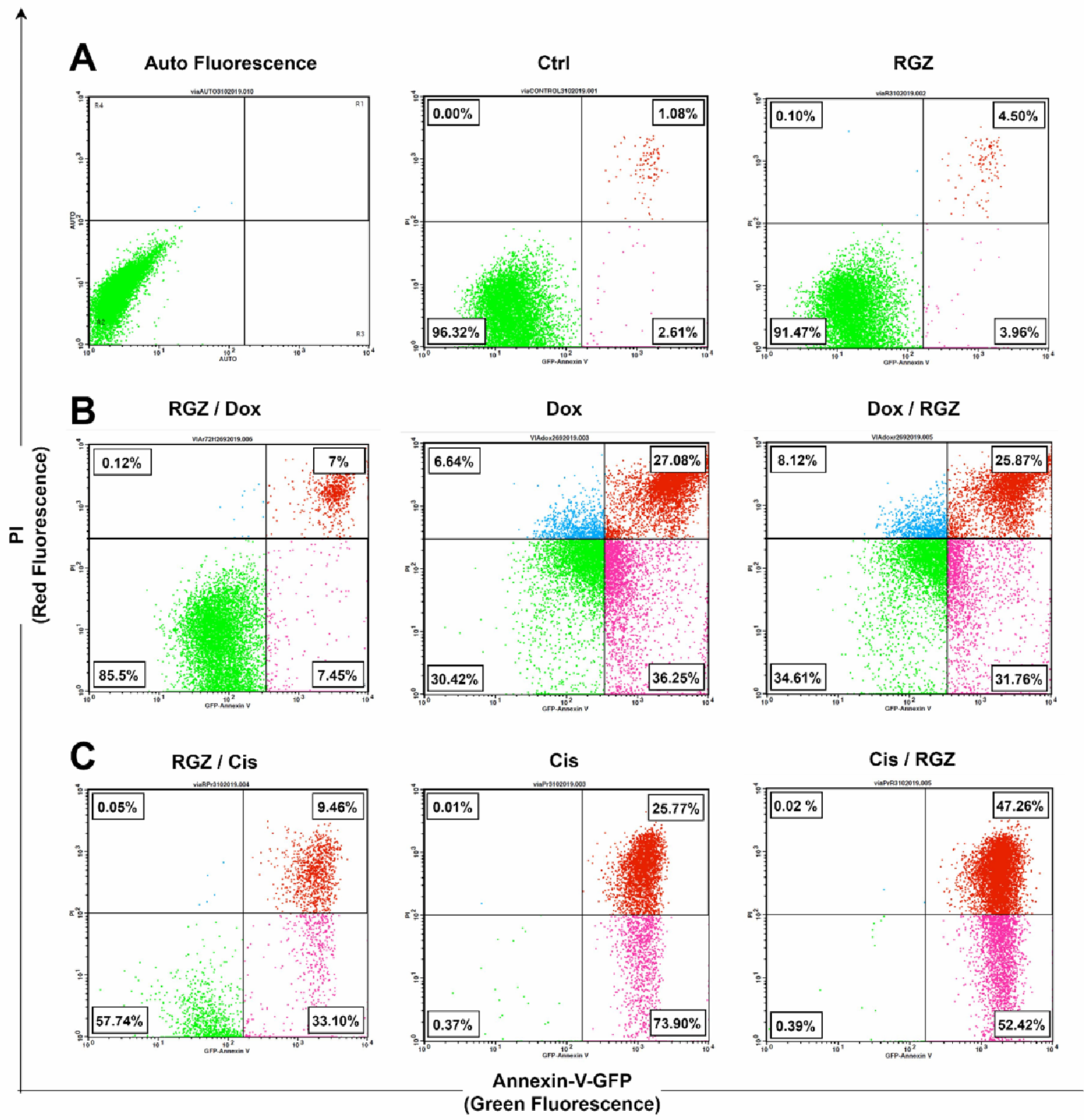

Fig. 4: Apoptosis/Necrosis assay of MCF-7 cells treated with various combinations of rosiglitazone (RGZ) and chemotherapeutic drugs using flow cytometry: (A) for control and RGZ-treated MCF-7 cells (left to right); (B) for doxorubicin (Dox), Dox/RGZ and RGZ/Dox combinations (left to right); and (C) for cisplatin (Cis), Cis/RGZ and RGZ/Cis combinations (left to right).

The preceded drug gave its cell cycle pattern to MCF-7 cells treated in the absence or presence of subsequently applied medication

To investigate the effect of previous treatments on the progression of the cell cycle, we performed cell cycle analysis with fluorescent DNA stain, propidium iodide (PI). Dox treatment caused a remarkable shift from G1 to $S$ phase, where cell proportion in the $S$ phase was $95.33 \%$ compared with $30.02 \%$ in control cells. Cells treated with Dox followed by RGZ exhibited similar behaviour. They showed an $\mathrm{S}$ phase arrest (87.61\%). Conversely, cell treatment with RGZ followed by Dox showed a small raise in $\mathrm{S}$ phase cell proportion with $(38.92 \%)$ and a decrease in G2/M phase cell proportion (0\%) compared with control cells $(2.6 \%)$. 
Our data showed similar results with $\mathrm{Cis}$ alone, and Cis followed RGZ treatment cells, which exhibited a slip in G0/G1 cell proportion (33.34\% and $23.9 \%$, respectively) and an increase in $\mathrm{S}$ phase cell proportion. In contrast, no significant difference in cell cycle was observed in RGZ alone, or RGZ followed by Cis compared with control (Fig. 5).

A

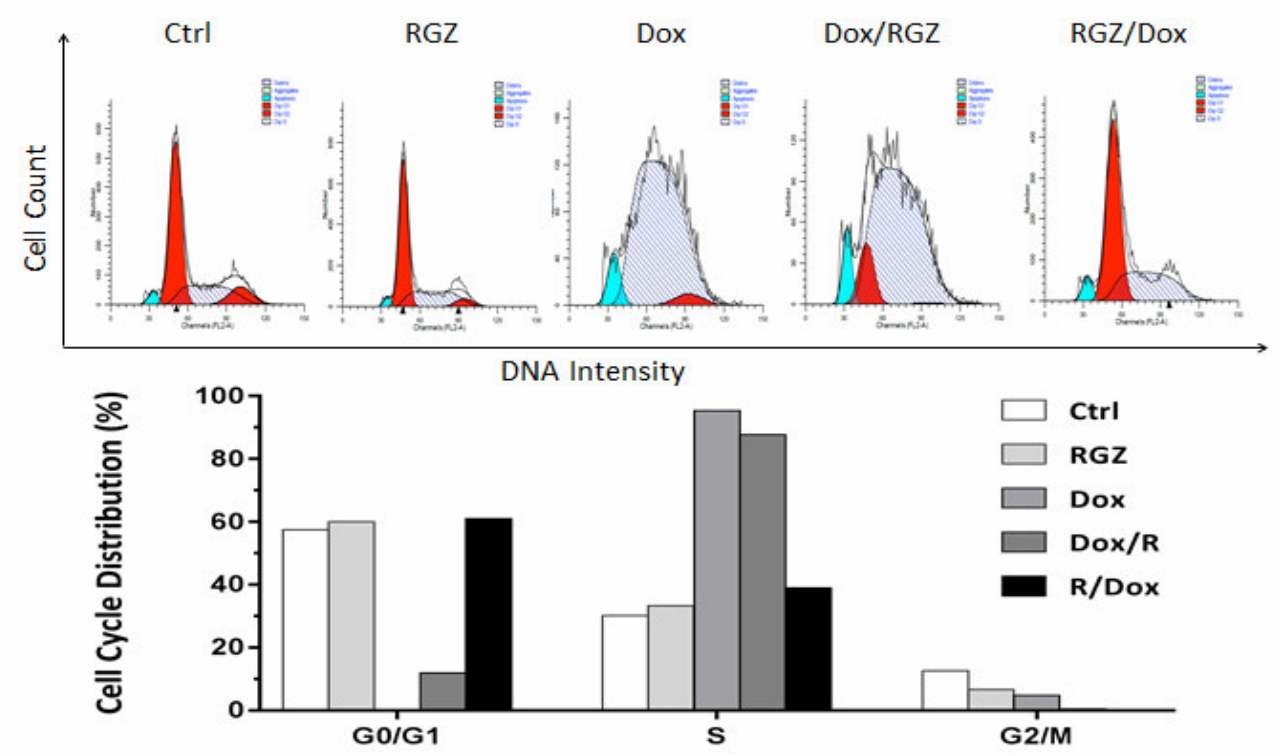

B

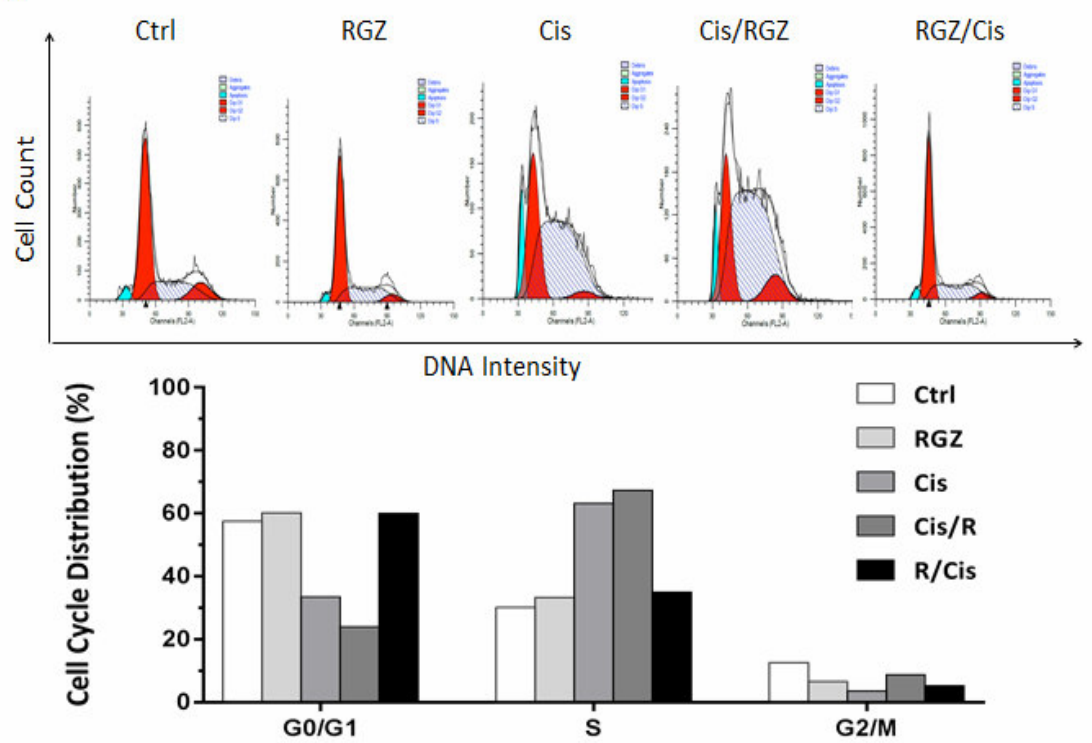

Fig. 5: Cell cycle analysis of MCF-7 cells treated with various combinations of rosiglitazone (RGZ) with Chemotherapeutic drugs using flow cytometry: (A) with doxorubicin (Dox) and (B) with cisplatin (Cis). The histogram of cell cycle distribution was generated from 10,000 events per sample. 
No lipid droplet accumulation was observed as a sequence of combinations application

To determine whether previous drug combinations led to lipid droplet accumulation in MCF-7 treated cells, we used the lipidspecific fluorescent dye, Nile blue. Neither control nor rosiglitazone-alone-treated cells nor combinations-treated cells showed fluorescence, indicating no lipid accumulation occurred under our experimental conditions. Interestingly, we observed few lipid droplets (not more than one to two droplets in a cell in approximately $15 \%$ of cells) in wells treated with Cis followed by RGZ.

\section{Discussion}

We found partial antagonism when applying drugs in chronological order: RGZ followed by chemotherapy regardless of chemotherapy type, its mechanism of action, or its concentration, which provides evidence for a shared mechanism that mediates the cellprotective effect of RGZ against cytotoxic therapeutic drugs. Noting that we added human recombinant insulin to the culture medium in our experiments, the protective effect might be due to the insulin-sensitizing action of RGZ as described by Djazayeri et al. ${ }^{16 \& 17}$ when worked on bone marrow recovery.

This type of experiments could not allow the identification of mechanisms underlying the effect of combination to be synergistic or antagonistic, which requires prolonged tests. However, our study may contribute to the filtering of compounds that are more likely to be effective in subsequent trials.

In our experiments, RGZ-Dox combinations did not show a synergism on MCF-7 cells. Patel et al. ${ }^{18}$ suggested a mechanism of action for Dox relying on its ability to increase ceramide production (a sphingolipid), which has raised recently as a second messenger in multiple signals transduction pathways, including the apoptosis pathway. This pathway is responsible for the apoptosis activity (Potentiation) effect of Dox. Other studies reported the role of ceramide in decreasing levels of PPAR $\gamma$ with a concurrent augmentation of its transcriptional activity. They mentioned that DOX-induced cell apoptosis is dependent on the PPAR $\gamma$ pathway ${ }^{19}$. That could explain the enhancement of RGZ of the Dox-inhibitory activity in relatively low concentrations. In contrast, we did not observe such inhibition in combinations containing higher Dox concentrations, with the underlying mechanism still unknown. Our results differ from those of Arif and his colleagues $^{20}$, who reported an increase in Doxtoxicity on MCF-7 breast cancer cell line when combined with RGZ.

Conversely, we found synergism when applying cytotoxic chemotherapeutic drugs (5FU and Cis), followed by RGZ on breast cancer cells. 5-FU induces the upregulation of the transient receptor potential cation channel subfamily V member 1 (TRPVI) gene in MCF7 cells, which, in turn, influences calcium entry and stimulates the production of intracellular ROS and activation of Poly (ADP-Ribose) Polymerase 1 (PARP1). In turn, RGZ elevation causes further activation of TRPV1 and an increase in calcium levels ${ }^{21}$.

Cis killed a higher percentage of MCF-7 cells (known to be sensitive to Cis) and probably induced cell cycle arrest in the $\mathrm{S}$ phase in more resistant subpopulations. This is consistent with researches showing that cell cycle progression arrests in Cis sensitive cell lines in the G2 phase, while cells enter apoptosis with high doses ${ }^{22 \& 23}$. Subsequent application of RGZ increased the proportion of both apoptotic and S-phase arrested cells, which implies a role of RGZ on cancer stem cell subpopulation associated with the MCF-7 cell line ${ }^{24}$.

Studies differed in assessing the role of PPAR $\gamma$ ligands in protecting non-cancerous cells from Cis-induced toxicity in a PPAR $\gamma$ dependent pathway, and its modulation of NF$\mathrm{KB}$ and TNF- $\alpha$ and oxidative stress in mice ${ }^{25 \& 26}$. Our results in breast cancer were consistent with Reddy at al. ${ }^{27}$ results showing a protective effect of TGZ on cells when applied to precede the chemotherapy on non-small cells lung cancer (NSCLC) cells, as they reported an antagonistic effect when treating cells with troglitazone (TGZ) (a PPAR $\gamma$ agonist) followed by Cis, unlike treating cells with Cis followed by TGZ, showing synergism resulted from Cis induction of $P P A R G$ expression which, in turn, enhances his effect later.

Finally, contrary to studies in which pioglitazone induces lipid droplets accumulation in MCF-7 cells ${ }^{2}$, we did not observe lipid droplets accumulation (as a 
marker of adipose-differentiation) in treated cells with any of the tested combinations except for the Cis/RGZ combination. It might be that $72 \mathrm{~h}$. treatment was not sufficient to translate the molecular signs into a marked phenotype and induce lipid synthesis and storage, or it could be that MCF-7 cells -known as having a relatively well-differentiated phenotype- were not the perfect model for studying this type of interaction.

The question remains: will our data invitro fit well with those in-vivo, considering the higher concentrations used of RGZ compared to typical plasma concentrations and its high protein binding property, which makes its free plasma concentrations much lower than invitro sufficient concentrations? Several factors may contribute to the RGZ activity, some of which relate to the nature of the tumor and its differentiation state, probably responsible for making it more sensitive or resistant to the differentiation effects of glitazones. In contrast, others relate to glitazones' nature and their ability to accumulate in some tissues in concentrations high enough to produce an aimed biological effect. This requires extensive studies of more diverse combinations of glitazones and chemotherapeutics to achieve perfect combinations.

\section{Conclusion}

Combinations containing the PPAR $\gamma$ ligand: RGZ with cytotoxic chemotherapeutic drugs (5-FU and Cis) showed sequencespecific synergism when RGZ followed chemotherapy. Whereas, when RGZ precedes chemotherapy, it blocked these cytotoxic effects. Nor additive or synergistic effect was not found when combining doxorubicin with RGZ.

All previous treatments did not lead to lipid droplet accumulation (as an indicator of RGZ-induced adipose differentiation) in treated MCF-7 cells regardless of drug concentrations and drug application sequence considering conditions and times experimented in our experiments.

We were limited as thiazolidinediones have many biologically overlapping effects and PPAR $\gamma$-dependent and independent targets, making it challenging to manage effects and interpret data. In parallel, MCF-7 represents a particular genetic, epigenetic, and phenotypic state and does not reflect the high heterogeneity of breast tumors in general. We can suggest that the application of combinations on other, and maybe lessdifferentiated, breast cancer cell lines or on invivo breast cancer models; and/or the use of treatment-times different from those applied in our study could lead to better effects and possibly, drug synergy.

\section{Acknowledgment}

The authors gratefully acknowledge Dr. Hossam Murad (AECS, Syria) for providing rosiglitazone and sharing his valuable experience and Mr. Abdulmunim Aljapawe for the analysis of flow cytometry data. Authors also extend their gratitude to Al-Assad University Hospital and Dr. Chadi Soukkarieh, Center for Epidemiological and Biological studies of Leishmania Parasites, for providing access to equipment used in this study.

\section{REFERENCES}

1- F. D. Cruz and I. Matushansky, "Solid tumor differentiation therapy - is it possible?", Oncotarget, 3 (5), 559-567 (2012).

2- E. Mueller, P. Sarraf, P. Tontonoz, R. M. Evans, K. J. Martin, M. Zhang, C. Fletcher, S. Singer and B. M. Spiegelman, "Terminal differentiation of human breast cancer through PPAR $\gamma$ ", Molecular Cell, 1 (3), 465-470 (1998).

3- W. P. Xu, X. Zhang and W. F. Xie, "Differentiation therapy for solid tumors", Journal of Digestive Diseases, 15 (4), 159-165 (2014).

4- A. A. Tran and V. Prasad, "Drug repurposing for cancer treatments: A wellintentioned, but misguided strategy", The Lancet Oncology, 21 (9), 1134-1136 (2020).

5- P. Nowak-Sliwinska, L. Scapozza and A. Ruiz I Altaba, "Drug repurposing in oncology: Compounds, pathways, phenotypes and computational approaches for colorectal cancer", Biochimica et Biophysica Acta (BBA) - Reviews on Cancer, 1871 (2), 434-454 (2019).

6- S. Kersten, B. Desvergne and W. Wahli, "Roles of PPARs in health and disease", Nature, 405 (6785), 421-424 (2000). 
7- C. Blanquicett, J. Roman and C. M. Hart, "Thiazolidinediones as anti-cancer agents", Cancer Therapy, 6 (A), 25-34 (2008).

8- S. Ryu, D. S. Kim, M. W. Lee, J. W. Lee, K. W. Sung, H. H. Koo and K. H. Yoo, "Anti-leukemic effects of PPAR $\gamma$ ligands", Cancer Letters, 418 (10-19 (2018).

9- M. H. Fenner and E. Elstner, "Peroxisome proliferator-activated receptor-gamma ligands for the treatment of breast cancer", Expert Opinion on Investigational Drugs, 14 (6), 557-568 (2005).

10- J. Zhou, W. Zhang, B. Liang, M. C. Casimiro, D. Whitaker-Menezes, M. Wang, M. P. Lisanti, S. Lanza-Jacoby, R. G. Pestell and C. Wang, "PPARgamma activation induces autophagy in breast cancer cells", The International Journal of Biochemistry \& Cell Biology, 41 (11), 2334-2342 (2009).

11- WHO, "Breast Cancer - Early Diagnosis and Screening", (2020).

12- T. C. Chou and P. Talalay, "Quantitative analysis of dose-effect relationships: the combined effects of multiple drugs or enzyme inhibitors", Advances in Enzyme Regulation, 22, 27-55 (1984).

13- T.-C. Chou and N. Martin, "CompuSyn for Drug Combinations and for General Dose-Effect Analysis", (2005).

14- A. Q. Abbady, A. Twair, B. Ali and H. Murad, "Characterization of annexin V fusion with the superfolder GFP in liposomes binding and apoptosis detection", Frontiers in Physiology, 8, 317-317 (2017).

15- Z. Darzynkiewicz, X. Huang and H. Zhao, "Analysis of cellular DNA content by flow cytometry", Current Protocols in Cytometry, 82 (1), 7.5.1-7.5.20 (2017).

16- K. Djazayeri, Z. Szilvássy, B. Peitl, J. Németh, L. Nagy, A. Kiss, B. Szabó and I. Benko, "Accelerated recovery of 5fluorouracil-damaged bone marrow after rosiglitazone treatment", European Journal of Pharmacology, 522 (1-3), 122-129 (2005).

17- K. Djazayeri, Z. Szilvássy, K. Benkő, B. Rózsa, B. Szabó, A. J. Szentmiklósi and I.
Benkö, "Effect of rosiglitazone, an insulin sensitizer, on myelotoxicity caused by repeated doses of 5-fluorouracil", Pharmacological Research, 53 (2), 156161 (2006).

18- A. G. Patel and S. H. Kaufmann, "How does doxorubicin work?", eLife, 1, e00387 (2012).

19- J. Wang, X. Lv, J. Shi and X. Hu, "Ceramide induces apoptosis via a peroxisome proliferator-activated receptor gamma-dependent pathway", Apoptosis: An International Journal on Programmed Cell Death, 11 (11), 20432052 (2006).

20- I. S. Arif, C. L. Hooper, F. Greco, A. C. Williams and S. Y. Boateng, "Increasing doxorubicin activity against breast cancer cells using PPAR $\gamma$-ligands and by exploiting circadian rhythms", British Journal of Pharmacology, 169 (5), 11781188 (2013).

21- H. A. Deveci and M. Nazıroğlu, "5Fluorouracil-induced mitochondrial oxidative cytotoxicity and apoptosis are increased in MCF-7 human breast cancer cells by TRPV1 channel activation but not Hypericum perforatum treatment", Molecular and Cellular Biochemistry, 439 (1-2), 189-198 (2018).

22- N. Hamaguchi, H. Hamada, S. Miyoshi, K. Irifune, R. Ito, T. Miyazaki and J. Higaki, "In-vitro and in-vivo therapeutic efficacy of the PPAR- $\gamma$ agonist troglitazone in combination with cisplatin against malignant pleural mesothelioma cell growth", Cancer Science, 101 (9), 1955-1964 (2010).

23- A. M. Heijink, M. Everts, M. E. Honeywell, R. Richards, Y. P. Kok, E. G. E. de Vries, M. J. Lee and M. van Vugt, "Modeling of cisplatin-induced signaling dynamics in triple-negative breast cancer cells reveals mediators of sensitivity", Cell Reports, 28 (9), 2345-2357.e2345 (2019).

24- J. S. Han and D. L. Crowe, "Tumor initiating cancer stem cells from human breast cancer cell lines", International Journal of Oncology, 34 (5), 1449-1453 (2009). 
25- M. M. Helmy, M. W. Helmy and M. M. El-Mas, "Additive Renoprotection by pioglitazone and fenofibrate against inflammatory, oxidative and apoptotic manifestations of cisplatin nephrotoxicity: Modulation by PPARs", PLoS ONE, 10 (11), e0142303 (2015).

26- O. Ozkaya, O. Yavuz, B. Can, M. Dilek, E. Savli, Y. Acikgoz, A. Bedir and T. Akpolat, "Effect of rosiglitazone on cisplatin-induced nephrotoxicity", Renal Failure, 32 (3), 368-371 (2010).
27- R. C. Reddy, A. Srirangam, K. Reddy, J. Chen, S. Gangireddy, G. P. Kalemkerian, T. J. Standiford and V. G. Keshamouni, "Chemotherapeutic drugs induce PPARgamma expression and show sequencespecific synergy with PPAR-gamma ligands in inhibition of non-small cell lung cancer", Neoplasia (New York, N.Y.), 10 (6), 597-603 (2008). 


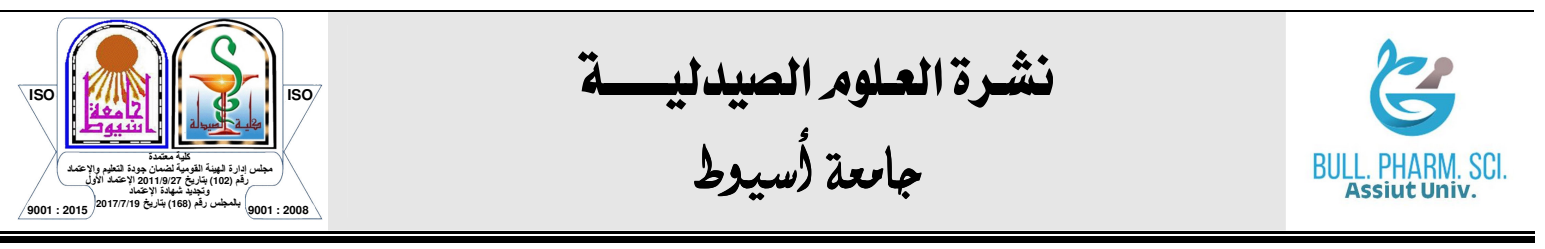

عقار الروزيجليتازون يظهر تتآزرًا خاصًا بالتسلسل مع أدوية العلاج الكيميائي

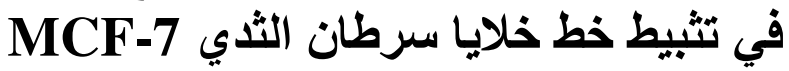

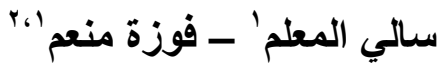

برنامج البيولوجيا الجزيئية والتكنولوجيا الحيوية ، قسم الكيمياء الحيويـة والأحياء الدقيقة ، كليـة الصيدلة ، جامعة دمشق ، دمشق ، سوريا

rقسم المختبرات السريرية ، مستشفى الأسد ، دمشق ، سوريات

أظهرت الدِّر اسـات مـا قبل السريريّة أنَّ لجـائن المستقبلات المفعَّلة بناشـر البيروكسيّة من النمط

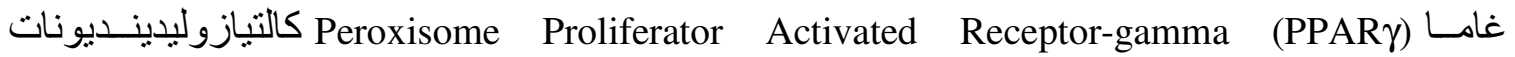

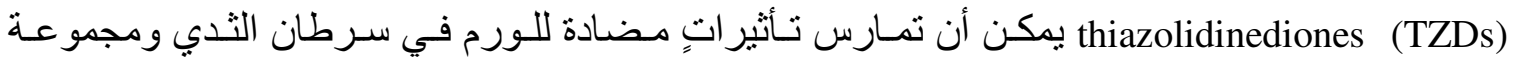

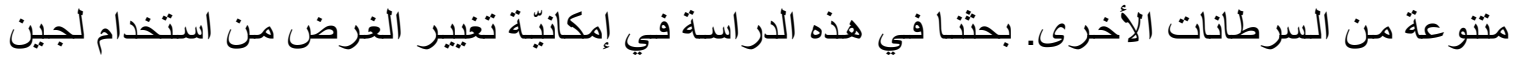

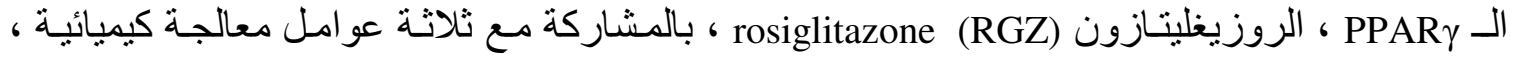

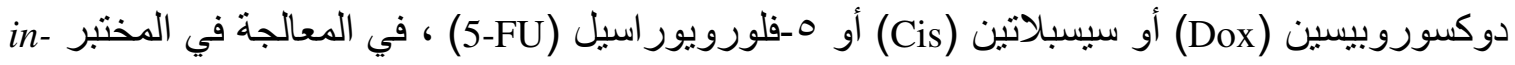

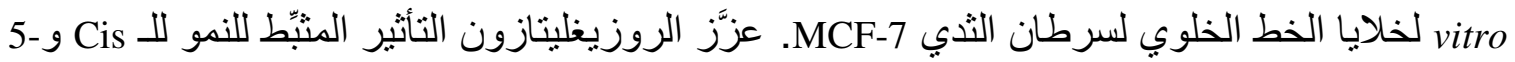

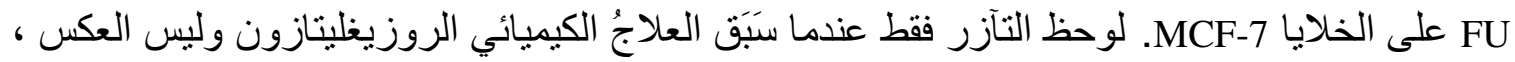

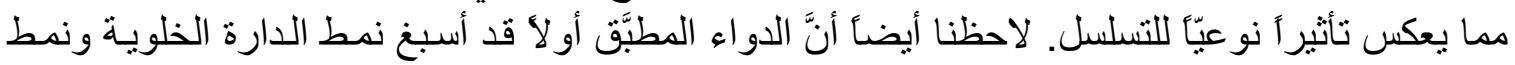

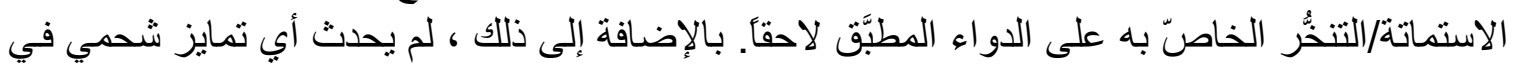

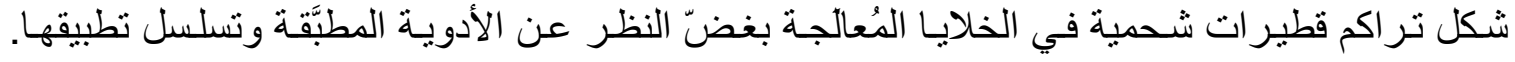

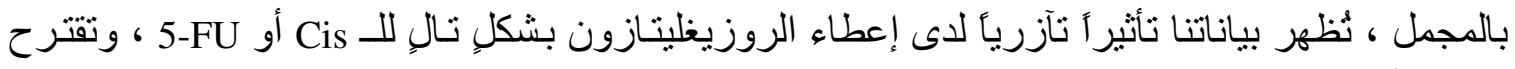
دورَ مثبِّطَّ للا RGZ على الجمهر ات الخلوية الفرعية للخلايا MCF-7 المقاومة للمعالجة الكيميائية. 